\title{
Differential Activation of ER Stress Signal Pathways Contributes to Palmitate-Induced Hepatocyte Lipoapoptosis
}

\author{
Xiaofang Zhao, Fuli Yao, Chunyan Zhang, Ying Cheng, Bin Xiao, Yuxiao Li, Chunyan Duan, \\ Youping Liu, Hong Li, Rongyang Dai ${ }^{*}$
}

Department of Biochemistry and Molecular Biology, Southwest Medical University, Luzhou, Sichuan, China

\section{Email address:}

zhaoxiaofang2099@163.com (Xiaofang Zhao), fuliyao@163.com (Fuli Yao), zcy_2004_116@163.com (Chunyan Zhang), 123964977@qq.com (Ying Cheng),xbwin@163.com (Bin Xiao), lyx452x@hotmail.com (Yuxiao Li), swallow0213@163.com (Chunyan Duan),lyp2507622@163.com (Youping Liu), lihong7188@163.com (Hong Li), dryrun2502@163.com (Rongyang Dai)

${ }^{*}$ Corresponding author

\section{To cite this article:}

Xiaofang Zhao, Fuli Yao, Chunyan Zhang, Ying Cheng, Bin Xiao, Yuxiao Li, Chunyan Duan, Youping Liu, Hong Li, Rongyang Dai. Differential Activation of ER Stress Signal Pathways Contributes to Palmitate-Induced Hepatocyte Lipoapoptosis. Cell Biology. Vol. 4, No. 1, 2016, pp. 1-8. doi: 10.11648/j.cb.20160401.11

Received: March 18, 2016; Accepted: March 28, 2016; Published: April 13, 2016

\begin{abstract}
Saturated free fatty acids-induced hepatocyte lipoapoptosis plays a pivotal role in non-alcoholic steatohepatitis. The activation of endoplasmic reticulum (ER) stress is involved in hepatocyte lipoapoptosis induced by the saturated free fatty acid palmitate (PA). However, the underlying mechanisms of the role of ER stress in hepatocyte lipoapoptosis remain largely unclear. In this study, we showed that PA and tunicamycin (Tun), a classic ER stress inducer, resulted in differential activation of ER stress pathways. Our data revealed that PA induced chronic and persistent ER stress response, but Tun induced acute and transient ER stress response. Compared with Tun treatment, PA induced much lower glucose-regulated protein 78 (GRP78), a central regulator of ER homeostasis, accumulation. It is noteworthy that GRP78 over-expression not only inhibited PA-induced ER stress but also decreased PA-induced apoptosis. Taken together, our data suggest that the differential activation of ER stress signal plays an important role in PA-induced hepatocyte lipoapoptosis. More detailed studies on the mechanisms of PA in repressing the accumulation of GRP78 will contribute to the understanding of molecular mechanisms of lipoapoptosis.
\end{abstract}

Keywords: ER Stress, Palmitate, Hepatocyte Lipoapoptosis, GRP78

\section{Introduction}

Non-alcoholic fatty liver disease (NAFLD) is a public health problem associated with the metabolic syndrome, such as obesity and insulin resistance [1]. Up to $30 \%$ of the population is afflicted by NAFLD and $5-10 \%$ of these patients develop non-alcoholic steatohepatitis (NASH), which can progress to cirrhosis and hepatocellular carcinoma [2,3]. Both obesity and insulin resistance are major risk factors for NASH and are characterized by an increase in circulating free fatty acids (FFAs) [4-6]. The excess serum FFAs in the context of insulin resistance are transported into hepatocyte by the fatty acid transporter. FFAs are esterified into neutral triglycerides in hepatocyte. However, surfeit of FFA is deleterious for hepatocyte. Saturated FFAs can induce hepatocyte apoptosis, a phenomenon termed lipoapoptosis [7-9]. Indeed, the lipoapoptosis of hepatocyte is a pathologic feature of NAFLD and correlates with disease severity [10-12]. Thus, the cellular and molecular mechanisms of hepatocyte lipoapoptosis are of biomedical interest.

The endoplasmic reticulum (ER) provides a contained environment for the synthesis and modification of membrane proteins and proteins destined to be secreted. Upon a number of biochemical, physiologic to pathologic stimuli, the ER protein processing system can be disrupted by disordered calcium $\left(\mathrm{Ca}^{2+}\right)$ homeostasis and redox status, which subsequently cause the accumulation of unfolded or 
misfolded proteins, a condition defined as ER stress. Conditions of ER stress lead to the onset of the unfolded protein response (UPR) [13-16]. The canonical UPR signaling pathways are mediated by three UPR transmembrane sensors: protein kinase RNA-like ER kinase (PERK), transcription factor activating transcription factor 6 (ATF6), and inositol-requiring enzyme 1 (IRE1) [17, 18]. In non-stressed situations, glucose-regulated protein 78 (GRP78) acts as a master regulator of the UPR through direct interaction with ATF6, PERK, and IRE1 and maintains them in an inactive form. Upon ER stress, GRP78 is titrated away by unfolded proteins, releasing the UPR sensors, which subsequently activates these sensors and initiates the activation and transduction of UPR signals. The UPR is a coordinated response to reduce the accumulation of misfolded proteins and restore ER function. If the efforts of UPR fail to restore ER homeostasis, the UPR will induce cell apoptosis to eliminate the stressed cells $[16,18]$.

Intracellular FFAs are esterified within the ER, and inundation of hepatocyte with FFA disturbs ER function, which resulting in ER stress response [19, 20]. The activation of UPR signal pathways have been well documented in NAFLD [19]. It has been postulated that ER may sense metabolic stresses associated with obesity and transmit signals that ultimately influence both pancreatic insulin secretion and hepatic insulin action. Since it has been reported that ER stress and inability of the UPR to reestablish ER homeostasis may be upstream components of lipotoxicity $[19,21]$, the mechanisms of ER stress in lipotoxicity are poorly defined.

In this study, we investigated the roles of ER stress in palmitate (PA, a 16 carbon FFA with no carbon-carbon double bonds, indicated 16:0)-induced hepatocyte lipoapoptosis. We found that PA caused ER stress as evidenced by increased X-box-binding protein-1 (XBP-1) mRNA splicing, increased expression of C/EBP homologous protein (CHOP) and GRP78. Interestingly, the pattern of PA-initiated ER stress is different from N-glycosylation inhibitor tunicamycin (Tun)-induced ER stress, and the differential activation of ER stress signal pathways plays an important role in hepatocyte lipoapoptosis.

\section{Materials and Methods}

\subsection{Chemicals and Antibodies}

Palmitate (PA), ER stress inducer tunicamycin (Tun, inhibitor of N-linked glycosylation), and ER stress inhibitor PBA were purchased from Sigma. Antibodies against phosphor-eIF2 $\alpha$ (Ser-51), PARP, and Myc-tag were purchased from Cell Signaling Technology. Antibodys against GRP78, and $\beta$-actin were purchased from Santa Cruz Biotechnology. GRP78 plasmid was purchased from Addgene.

\subsection{Cell Culture and Transfection}

Human hepatocellular carcinoma cell line SMMC-7721 was cultured in Dulbecco's modified Eagle's medium containing $10 \%$ fetal bovine serum and $1 \%$ penicillin/streptomycin antibiotics. Cells were maintained at $37^{\circ} \mathrm{C}$ in a humidified incubator containing $5 \% \mathrm{CO}_{2}$ and $95 \%$ air. Transfection of vectors for the expression of GFP and GRP78 were performed using Lipofectamine 2000 (Invitrogen, Carlsbad, CA, USA) according to the manufacturer's manuals.

\subsection{Fatty Acid Treatment}

PA was dissolved in isopropanol at a concentration of 200 $\mathrm{mM}$. PA was added to DMEM containing $1 \%$ bovine serum albumin to obtain a physiologic ratio between bound and unbound FFA in the media. The concentration of PA used in the experiments was $500 \mu \mathrm{M}$ and was similar to the fasting total FFA plasma concentrations observed in human with non-alcoholic steatohepatitis.

\subsection{Western Blot Analysis}

The procedure for Western blot has been described in detail previously [22-24]. Briefly, cells were lysed in Triton lysis buffer (20 mM Tris, pH 7.4, $137 \mathrm{mM} \mathrm{NaCl,} \mathrm{10 \%} \mathrm{glycerol,} \mathrm{1 \%}$ Triton X-100, 2 mM EDTA, $1 \mathrm{mM}$ PMSF, $10 \mathrm{mM}$ sodium fluoride, $5 \mathrm{mg} / \mathrm{ml}$ of aprotinin, $20 \mathrm{mM}$ leupeptin, and $1 \mathrm{mM}$ sodium orthovanadate) and centrifuged at 12,000 $\mathrm{g}$ for 15 minutes. Protein concentrations were measured using the BCA assay. Protein samples were denatured with $4 \times$ SDS-loading buffer (200 mM Tris, pH 6.8, 8\% SDS, $400 \mathrm{mM}$ DTT, 0.4\% bromphenol blue, $40 \%$ glycerol) at $100{ }^{\circ} \mathrm{C}$ for 5 minutes and subjected to standard SDS-PAGE and Western blot analysis.

\subsection{Apoptosis Analysis}

Apoptosis was detected using the annexin V-FITC apoptosis detection kit (Invitrogen) according to the manufacturer's manual. The experiments were repeated three times.

\subsection{Reverse Transcription Reaction}

Total RNA was prepared as detailed previously [22, 24, 25]. Reverse transcription was performed with $0.2 \mu \mathrm{g}$ of isolated total RNA using the M-MLV reverse transcriptase kit (Promega). 18S was used as a normalization control. PCR products were run on 2-4\% agarose gel. The gel images were digitally captured with a SynGene gel documentation system and analyzed with the Genetools analysis software (Syngene, Frederick, MD, USA) as previously described [22, 24, 25].

The primers used in this study:

18S-forward GGGAGGTAGTGACGAAAAAT,

18S-reverse ACCAACAAAATAGAACCGCG;

XBP1-forward CCTTGTAGTTGAGAACCAGG,

XBP1-reverse GGGGCTTGGTATATATGTGG;

GRP78-forward ATCACGCCGTCCTATGTCGC,

GRP78-reverse TCTCCCCCTCCCTCTTATCC;

GADD153-forward AGTCATTGCCTTTCTCTTCG,

GADD153-reverse GGTGCAGATTCACCATTCGG.

\subsection{Statistical Analysis}

Results were expressed as the mean \pm S.D. Statistical analysis was performed using Student's t test. $p<0.05$ was considered statistically significant. 

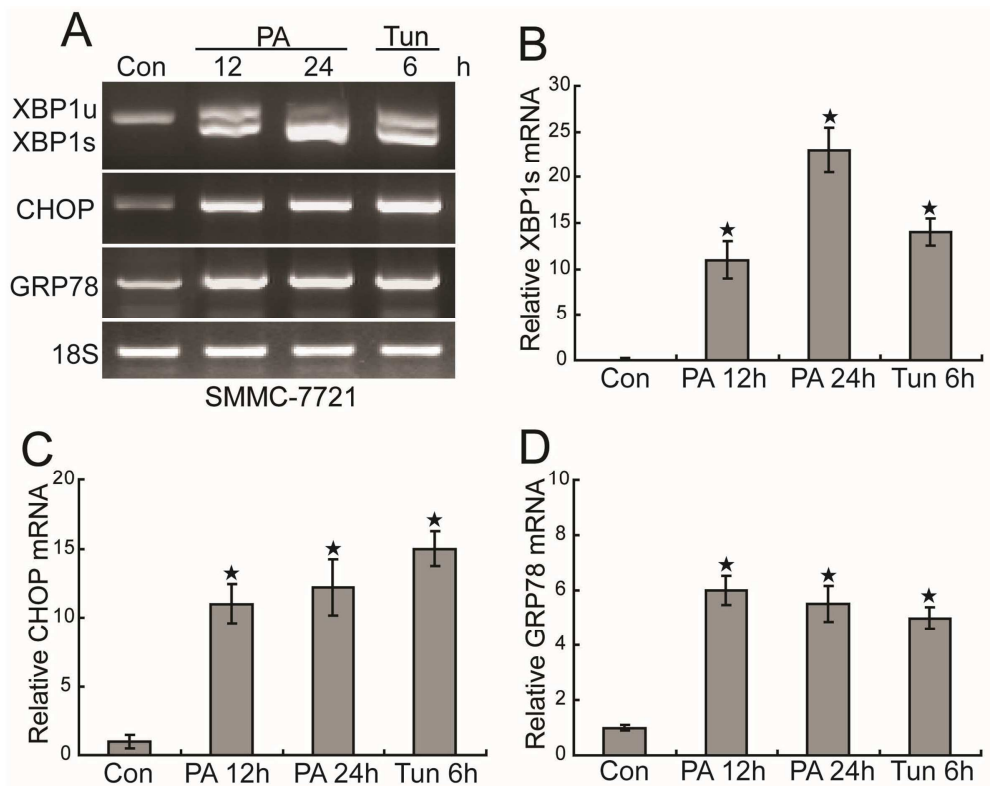

Figure 1. PA induces ER stress. (A) SMMC-7721 cells were treated with palmitate (PA, $500 \mu \mathrm{M})$ or tunicamycin (Tun, $2.5 \mathrm{mg} / \mathrm{ml}$ ) for indicated periods and total $R N A$ were analyzed by RT-PCR. $(B-D)$ The $m R N A$ values of spliced XBP1 $(X B P 1 s, B), C H O P(C)$ and GRP78 (D) were normalized to $18 S r R N A$ content. Data are presented as mean values $\pm S D$ of three measurements; bars, $S E$. *Significantly different from control value.

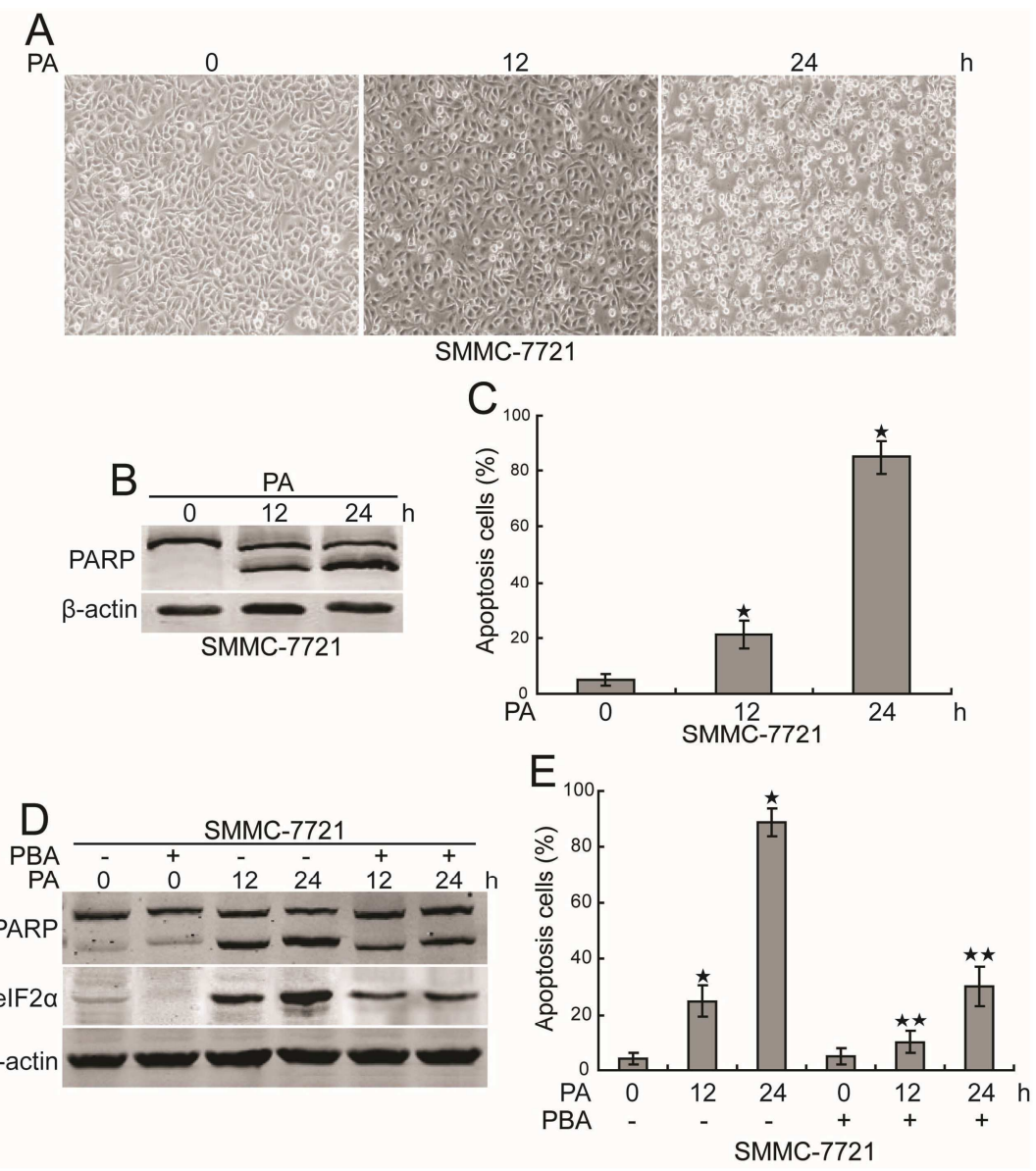

Figure 2. ER stress is involved in PA-induced apoptosis. (A-C) SMMC-7721 cells were treated with palmitate (PA, $500 \mu M)$ for indicated periods and apoptosis was detected by morphological examination (A), Western blot analysis (B) and annexin V-FITC apoptosis detection kit (C). Data are presented as mean values \pm $S D$ of three measurements; bars, SE. *Significantly different from control value. (D, E) SMMC-7721 cells were treated with palmitate (PA, $500 \mu M$ ) for indicated periods with or without ER stress inhibitor PBA (1 mM) pretreatment for 1 hour and apoptosis was detected by Western blot analysis (D) and annexin V-FITC apoptosis detection kit (E). Data are presented as mean values $\pm S D$ of three measurements; bars, SE. *Significantly different from control value. **significantly different from *value. 


\section{Results}

\subsection{PA Initiates ER Stress}

To investigate the potential role of PA in the induction of ER stress in hepatocyte, we first examined the ER stress induction effects of PA in SMMC-7721 cells. The mRNA levels of ER stress markers, including XBP1, CHOP, and GRP78, were evaluated by RT-PCR from PA treated SMMC-7721 cells. We found that PA treatment markedly elevated both the mRNA levels of GRP78 and CHOP, and induced XBP1 mRNA splicing, an indicator of UPR activation, in SMMC-7721 cells (Figure 1A). Tun-treated SMMC-7721 cells were used as positive control. For quantitative analysis, the mRNA levels of spliced XBP1, GRP78, and CHOP were normalized to the corresponding $18 \mathrm{~S}$ rRNA levels (Figure 1B-D). These data suggest that PA can initiate ER stress response in hepatocyte.

\subsection{ER Stress Is Involved in PA-initiated Apoptosis}

To investigate the potential role of PA in the induction of apoptosis in hepatocyte, we investigated the cytotoxicity effects of PA in SMMC-7721 cells. The results showed that PA treatment exerts obviously cytotoxicity in SMMC-7721 cells in a time-dependent manner (Figure 2A). In order to confirm the role of PA in inducing SMMC-7721 cells apoptosis, cleavage of poly (ADP-ribose) polymerase (PARP) was detected by Western blot analysis. Western blot analysis revealed that PA treatment induced the cleavage of PARP in SMMC-7721 cells in a time-dependent manner (Figure 2B). Furthermore, Annexin V-FITC staining (Figure 2C) confirmed that PA treatment can induce SMMC-7721 cells apoptosis in a time-dependent manner. Thus, these data suggest that PA can initiate apoptosis of hepatocyte.

To make sure whether ER stress is involved in PA-mediated apoptosis, ER stress inhibitor PBA was used to block the activation of ER stress. The results showed that PBA pre-incubation not only inhibited the phosphorylation of eIF $2 \alpha$ an indicator of UPR activation, but also obviously decreased the apoptosis of SMMC-7721 cells upon PA treatment (Figure 2D \& E). Taken together, these data suggest that ER stress plays an important role in PA-induced SMMC-7721 cells apoptosis.

Considering that ER stress plays a pivotal role in PA-induced SMMC-7721 cells apoptosis, we examined whether ER stress can induce apoptosis in SMMC-7721 cells upon Tun treatment. The data showed that SMMC-7721 cells were relatively resistant to ER stress-induced apoptosis triggered by Tun treatment for a relatively long time $(<15 \%$ apoptotic cells at $36 \mathrm{~h}$ ) (Figure $3 \mathrm{~A} \mathrm{\&} \mathrm{B).} \mathrm{However,} \mathrm{PA}$ treatment efficiently killed SMMC-7721 cells by induction of apoptosis at $24 \mathrm{~h}$ (Figure $3 \mathrm{~A} \& \mathrm{~B})$.

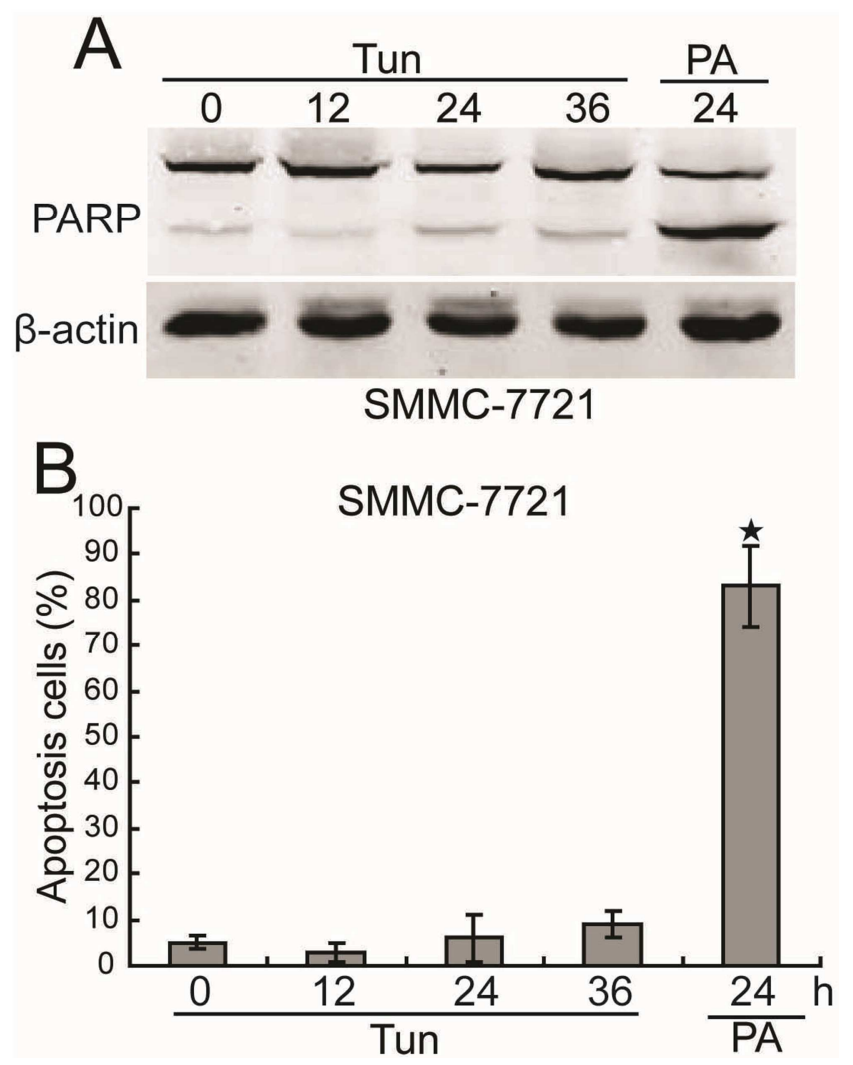

Figure 3. SMMC-7721 cells are resistant to ER stress-induced apoptosis. (A, B) SMMC-7721 cells were treated with tunicamycin (Tun, $2.5 \mathrm{mg} / \mathrm{ml}$ ) or palmitate $(P A, 500 \mu M)$ for indicated periods and apoptosis was detected by Western blot analysis (A) and annexin V-FITC apoptosis detection kit (B). Data are presented as mean values $\pm S D$ of three measurements; bars, $S E$. * Significantly different from control value.

\subsection{PA Differently Activates ER Stress Signal Pathways}

As ER stress induced by PA and Tun plays different roles in the induction of apoptosis, we investigated whether PA and Tun can differently activate ER stress signal pathways. The results showed that the mRNA levels of spliced XBP1 began accumulating within $3 \mathrm{~h}$ and decreased after $12 \mathrm{~h}$ in SMMC-7721 cells, when exposed to Tun (Figure 4A). In contrast, the up-regulating of spliced XBP1 mRNA levels can't be observed within $6 \mathrm{~h}$ in SMMC-7721 cells upon PA treatment (Figure 4A). However, PA-induced up-regulating of spliced XBP1 mRNA levels sustained more than $24 \mathrm{~h}$ (Figure 4A). Upon PA and Tun treatment, no obvious difference of CHOP mRNA levels was observed in SMMC-7721 cells (Figure 4A). Moreover, Tun-induced up-regulating of GRP78 mRNA levels are not only higher but also earlier than that of $\mathrm{PA}$ in SMMC-7721 cells (Figure 4A). For quantitative analysis, the mRNA levels of spliced XBP1, CHOP, and GRP78 were normalized to the corresponding 18S rRNA levels (Figure 4B-D). Together, these data indicate that PA and Tun initiate ER stress pathways activation with different kinetics specifically. 

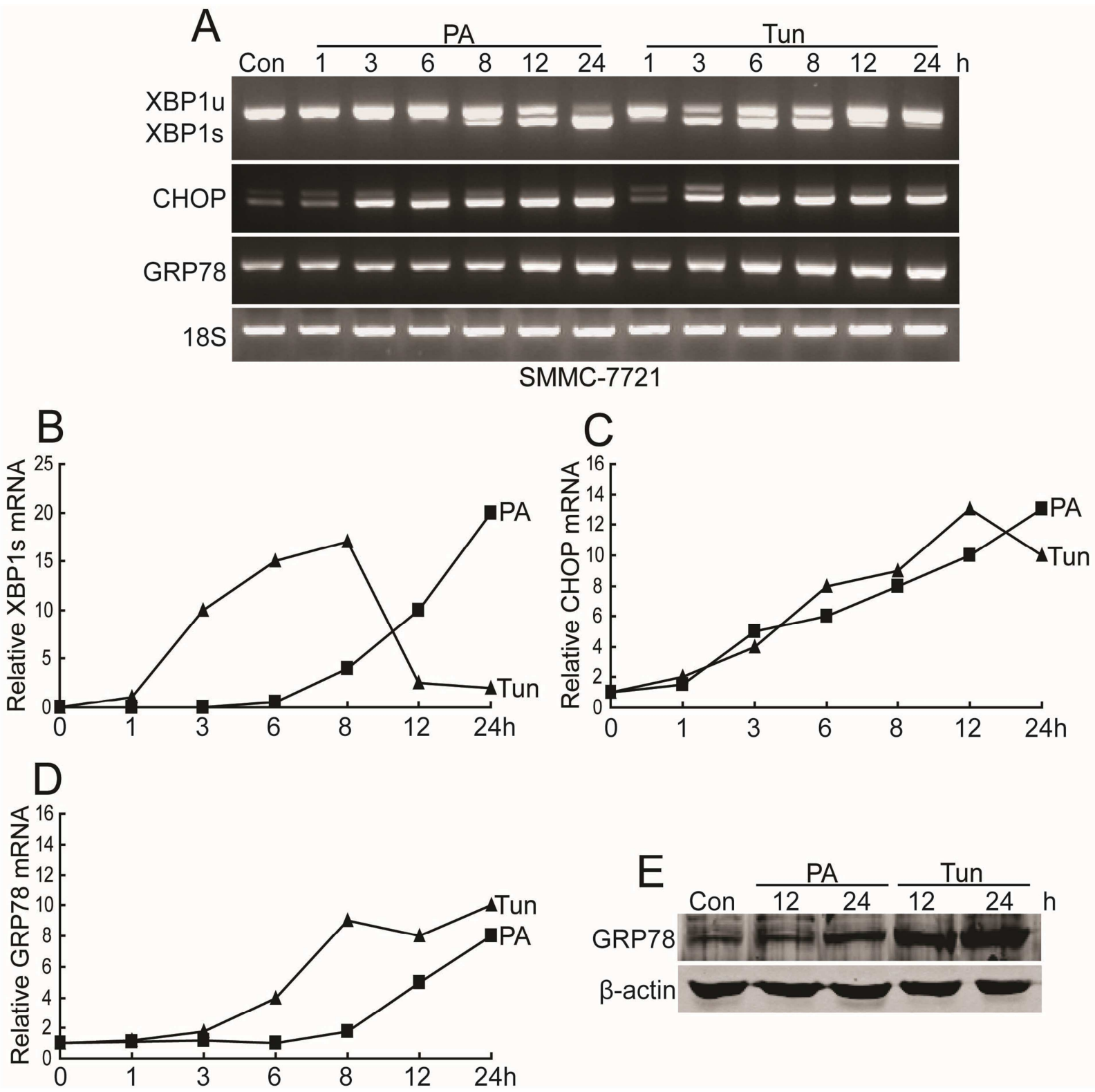

Figure 4. PA and Tun activate ER stress pathways with different kinetics. (A) SMMC-7721 cells were treated with palmitate (PA, $500 \mu M)$ or tunicamycin (Tun, $2.5 \mathrm{mg} / \mathrm{ml}$ ) for indicated periods and total RNA were analyzed by RT-PCR. (B-D) The $\mathrm{mRNA}$ values of spliced XBP1 (XBP1s, B), CHOP(C), GRP78 (D) were normalized to $18 S \mathrm{~S} r N A$ content. Data are presented as mean values $\pm S D$ of three measurements. (E) SMMC-7721 cells were treated with palmitate (PA, $500 \mu M$ ) or tunicamycin (Tun, $2.5 \mathrm{mg} / \mathrm{ml}$ ) for indicated periods and cell lysates were analyzed for the levels of indicated proteins.

\subsection{GRP78 Inhibits PA-induced Apoptosis}

Since the accumulation of GRP78 is inhibited in SMMC-7721 cells upon PA treatment, we therefore investigated whether GRP78 over-expression can protect SMMC-7721 cells from PA-induced apoptosis. The Western blot data showed that GRP78 over-expression obviously inhibited PA-mediated SMMC-7721 cells apoptosis (Figure 5A). The effects of GRP78 on apoptosis inhibition in PA-treated SMMC-7721 cells were reconfirmed by annexin V-FITC apoptosis analysis (Figure 5B). To evaluate whether GRP78 over-expression can decrease PA-induced ER stress response, spliced XBP1 mRNA levels were measured in GRP78 over-expressed SMMC-7721 cells and the control cells. As shown in Figure 5C, GRP78 over-expression decreased the induction of spliced XBP1 mRNA in PA-treated SMMC-7721 cells. Taken together, these data indicate that GRP78 deficiency plays an important role in PA-mediated apoptosis.

Since GRP78 plays a pivotal role in protecting cells from ER stress-induced apoptosis, we therefore examined the induction of GRP78 protein upon PA and Tun treatment in SMMC-7721 cells. As shown in Figure 4E, GRP78 protein levels induced by PA were much lower than that of Tun in SMMC-7721 cells. Thus, it is suggested that differential GRP78 accumulation might be a reason for the different roles of PA and Tun-induced ER stress in apoptosis inducing. 


\section{Figure 5}

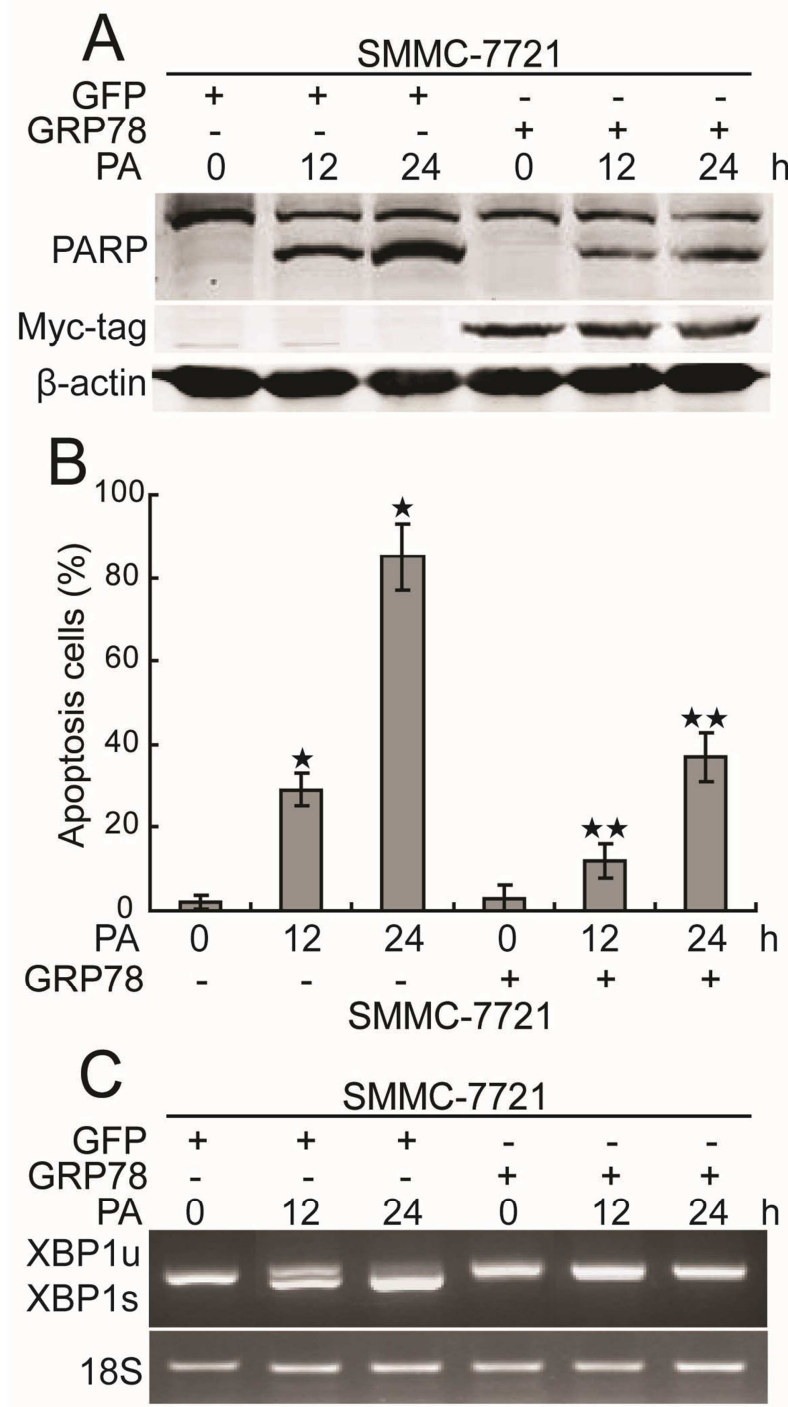

Figure 5. GRP78 inhibits PA-induce ER stress and apoptosis. (A, B) After transient transfection with GFP or Myc-GRP78 constructs for $24 \mathrm{~h}$, $S M M C-7721$ cells were treated with palmitate $(P A, 500 \mu M)$ for indicated periods and apoptosis was detected by Western blot analysis (A) and annexin $V$-FITC apoptosis detection kit (B). Data are presented as mean values $\pm S D$ of three measurements; bars, SE. *Significantly different from control value. **significantly different from *value. (C) After transient transfection with GFP or Myc-GRP78 constructs for 24 h, SMMC-7721 cells were treated with palmitate $(P A, 500 \mu \mathrm{M})$ for indicated periods and total $R N A$ were analyzed by RT-PCR.

\section{Discussion}

PA is one of the most common fatty acids in human diets as well as in animal and human fat tissue. PA is also the end product of de novo fatty acid synthesis and is generated in large quantity in subjects on a low-fat diet. The toxic effects of PA on cell viability have been reported in several cell types [26-30]. Although PA is implicated in the induction of apoptosis, it remains largely unclear how PA exerts its pro-apoptotic action. The results of the present study provide mechanistic insights regarding the pro-apoptotic effects of ER stress during PA-induced lipoapoptosis. Our data reveal that PA initiates ER stress and induces apoptosis in SMMC-7721 cells in part through decreasing the induction of GRP78, which results in persistent ER stress activation.

It has been reported that PA can initiate ER stress response in various cell types [29-32]. We firstly detected the potential role of PA in the induction of ER stress in SMMC-7721 cells. In agreement with these reports, we found that PA initiates ER stress in SMMC-7721 cells, evidenced by increased mRNA levels of spliced XBP1, CHOP and GRP78. Our data indicate that at least two of the three major UPR signaling pathways, IRE1/XBP1 and PERK/CHOP were altered by PA. It has been suggested that ER stress is involved in PA-induced apoptosis $[12,19,20,31]$. Based on the data that blocking ER stress inhibited PA-induced apoptosis in SMMC-7721 cells, we suggest that ER stress promotes PA-induced apoptosis in hepatocyte.

The UPR is an adaptive response that increases cell survival under ER stress conditions [16, 17]. However, if ER stress is persistent and the stress can't be resolved, the UPR signaling switches from pro-survival to pro-apoptotic mode and the cells are destroyed by apoptosis [22, 24, 33, 34]. Since SMMC-7721 cells are more resistant to ER stress-induced apoptosis than that of PA, we suggest that the activation pattern of PA-induced ER stress signal might be different from classic ER stress inducer Tun-induced ER stress signal. This hypothesis is supported by our data, which demonstrated PA and Tun activated UPR pathways with different kinetics. In the present study, we found that Tun-induced spliced XBP1 mRNA accumulation decreased within $12 \mathrm{~h}$. However, the PA-induced the accumulation of spliced XBP1 mRNA sustained high levels more than $24 \mathrm{~h}$. As the induction of spliced XBP1 mRNA is a potential biomarker of the degree of ER stress response $[35,36]$, it is reasonable to suggest that PA-induced ER stress is persistent, but Tun-induced ER stress is transient. The difference of lasting time between PA and Tun-induced ER stress response might be a major reason for the different effects of PA and Tun-induced ER stress in apoptosis induction.

How does PA induce persistent ER stress? As a central regulator of ER homeostasis and the UPR, GRP78 plays multiple roles in protein folding, protein quality control, maintaining ER homeostasis, and controlling of the activation of transmembrane ER stress sensors [37-39]. Considering that PA-induced up-regulation of GRP78 mRNA is lower than that of Tun, it seems that PA can't efficiently induce the accumulation of GRP78. This speculation was demonstrated by the findings that the accumulation of GRP78 protein was much lower than that of Tun. In our case, it is reasonable to speculate that PA-induced ER stress is persistent, and the stressed cells can't recover from ER stress duo to the deficient induction of GRP78. It's notable that GRP78 over-expression not only decreased the degree of PA-induced ER stress, but also inhibited PA-mediated apoptosis. These data reconfirm that the deficient induction of GRP78 exerts the pro-apoptotic effect of PA-induced ER stress. 


\section{Conclusion}

The current study demonstrates that ER stress plays an essential role in PA-induced hepatocyte apoptosis. Our data provide mechanistic insights regarding the link between ER stress activation and FFA-mediated lipotoxicity. The ability of PA to inhibit GRP78 induction exerts the pro-apoptotic effect of PA-induced ER stress. More detailed studies on the mechanisms of PA in repressing the accumulation of GRP78 will contribute to the understanding of molecular mechanisms of lipoapoptosis.

\section{Acknowledgements}

This work was funded by grants from the National Natural Science Foundation of China (81472312), Sichuan Province Science Foundation for Youths (2013JQ0045), New Century Excellent Talents in University Grant (NCET-11-1058), Innovation Team of Education Department of Sichuan Province (16TD0021), Luzhou City-Southwest Medical University Foundation (2013LZLY-J06), and Sichuan Province-Luzhou City-Southwest Medical University Foundation (14JC0082, 14JC0038, 14ZC0070).

\section{References}

[1] Yki-Jarvinen H. Non-alcoholic fatty liver disease as a cause and a consequence of metabolic syndrome. Lancet Diabetes Endocrinol 2014; 2: 901-910.

[2] Adams LA, Lymp JF, St Sauver J, Sanderson SO, Lindor KD, Feldstein A, et al. The natural history of nonalcoholic fatty liver disease: a population-based cohort study. Gastroenterology 2005; 129: 113-121.

[3] Browning JD, Szczepaniak LS, Dobbins R, Nuremberg P, Horton JD, Cohen JC, et al. Prevalence of hepatic steatosis in an urban population in the United States: impact of ethnicity. Hepatology 2004; 40: 1387-1395.

[4] Sanyal AJ, Campbell-Sargent C, Mirshahi F, Rizzo WB, Contos MJ, Sterling RK, et al. Nonalcoholic steatohepatitis: association of insulin resistance and mitochondrial abnormalities. Gastroenterology 2001; 120: 1183-1192.

[5] Kusminski CM, Shetty S, Orci L, Unger RH, Scherer PE. Diabetes and apoptosis: lipotoxicity. Apoptosis 2009; 14: 1484-1495.

[6] Watson ML, Macrae K, Marley AE, Hundal HS. Chronic effects of palmitate overload on nutrient-induced insulin secretion and autocrine signalling in pancreatic MIN6 beta cells. PLoS One 2011; 6: e25975.

[7] Unger RH, Orci L. Lipoapoptosis: its mechanism and its diseases. Biochim Biophys Acta 2002; 1585: 202-212.

[8] Ibrahim SH, Akazawa Y, Cazanave SC, Bronk SF, Elmi NA, Werneburg NW, et al. Glycogen synthase kinase-3 (GSK-3) inhibition attenuates hepatocyte lipoapoptosis. J Hepatol 2011; 54: 765-772.

[9] Wang JW, Wan XY, Zhu HT, Lu C, Yu WL, Yu CH, et al. Lipotoxic effect of $\mathrm{p} 21$ on free fatty acid-induced steatosis in L02 cells. PLoS One 2014; 9: e96124.
[10] Malhi H, Gores GJ. Molecular mechanisms of lipotoxicity in nonalcoholic fatty liver disease. Semin Liver Dis 2008; 28: 360-369.

[11] Feldstein AE, Gores GJ. Apoptosis in alcoholic and nonalcoholic steatohepatitis. Front Biosci 2005; 10: 3093-3099.

[12] Akazawa Y, Cazanave S, Mott JL, Elmi N, Bronk SF, Kohno S, et al. Palmitoleate attenuates palmitate-induced Bim and PUMA up-regulation and hepatocyte lipoapoptosis. J Hepatol 2010; 52: 586-593.

[13] Kaufman RJ. Stress signaling from the lumen of the endoplasmic reticulum: coordination of gene transcriptional and translational controls. Genes Dev 1999; 13: 1211-1233.

[14] Mori K. Tripartite management of unfolded proteins in the endoplasmic reticulum. Cell 2000; 101: 451-454.

[15] Harding HP, Calfon M, Urano F, Novoa I, Ron D. Transcriptional and translational control in the Mammalian unfolded protein response. Annu Rev Cell Dev Biol 2002; 18: 575-599.

[16] Rutkowski DT, Kaufman RJ. A trip to the ER: coping with stress. Trends Cell Biol 2004; 14: 20-28.

[17] Ron D, Walter P. Signal integration in the endoplasmic reticulum unfolded protein response. Nat Rev Mol Cell Biol 2007; 8: 519-529.

[18] Schroder M, Kaufman RJ. The mammalian unfolded protein response. Annu Rev Biochem 2005; 74: 739-789.

[19] Wei Y, Wang D, Topczewski F, Pagliassotti MJ. Saturated fatty acids induce endoplasmic reticulum stress and apoptosis independently of ceramide in liver cells. Am J Physiol Endocrinol Metab 2006; 291: E275-281.

[20] Gregor MF, Hotamisligil GS. Thematic review series: Adipocyte Biology. Adipocyte stress: the endoplasmic reticulum and metabolic disease. J Lipid Res 2007; 48: 1905-1914.

[21] Guo W, Wong S, Xie W, Lei T, Luo Z. Palmitate modulates intracellular signaling, induces endoplasmic reticulum stress, and causes apoptosis in mouse 3T3-L1 and rat primary preadipocytes. Am J Physiol Endocrinol Metab 2007; 293: E576-586.

[22] Dai RY, Chen Y, Fu J, Dong LW, Ren YB, Yang GZ, et al. p28GANK inhibits endoplasmic reticulum stress-induced cell death via enhancement of the endoplasmic reticulum adaptive capacity. Cell Res 2009; 19: 1243-1257.

[23] Chen R, Li J, Feng CH, Chen SK, Liu YP, Duan CY, et al c-Met function requires $\mathrm{N}$-linked glycosylation modification of pro-Met. J Cell Biochem 2013; 114: 816-822.

[24] Dai R, Li J, Fu J, Chen Y, Yu L, Zhao X, et al. Disturbance of $\mathrm{Ca} 2+$ homeostasis converts pro-Met into non-canonical tyrosine kinase p190MetNC in response to endoplasmic reticulum stress in MHCC97 cells. J Biol Chem 2012; 287: 14586-14597.

[25] Dai RY, Zhao XF, Li JJ, Chen R, Luo ZL, Yu LX, et al. Implication of transcriptional repression in compound C-induced apoptosis in cancer cells. Cell Death Dis 2013; 4: e883. 
[26] Miller TA, LeBrasseur NK, Cote GM, Trucillo MP, Pimentel DR, Ido Y, et al. Oleate prevents palmitate-induced cytotoxic stress in cardiac myocytes. Biochem Biophys Res Commun 2005; 336: 309-315.

[27] Listenberger LL, Ory DS, Schaffer JE. Palmitate-induced apoptosis can occur through a ceramide-independent pathway. J Biol Chem 2001; 276: 14890-14895.

[28] Manukyan L, Ubhayasekera SJ, Bergquist J, Sargsyan E, Bergsten P. Palmitate-induced impairments of beta-cell function are linked with generation of specific ceramide species via acylation of sphingosine. Endocrinology 2014: en20141467.

[29] Rojas C, Pan-Castillo B, Valls C, Pujadas G, Garcia-Vallve S, Arola L, et al. Resveratrol Enhances Palmitate-Induced ER Stress and Apoptosis in Cancer Cells. PLoS One 2014; 9: e113929.

[30] Simon-Szabo L, Kokas M, Mandl J, Keri G, Csala M. Metformin attenuates palmitate-induced endoplasmic reticulum stress, serine phosphorylation of IRS-1 and apoptosis in rat insulinoma cells. PLoS One 2014; 9: e97868.

[31] Park M, Sabetski A, Kwan Chan Y, Turdi S, Sweeney G. Palmitate induces ER stress and autophagy in H9c2 cells: implications for apoptosis and adiponectin resistance. J Cell Physiol 2015; 230: 630-639.

[32] Leamy AK, Egnatchik RA, Shiota M, Ivanova PT, Myers DS,
Brown HA, et al. Enhanced synthesis of saturated phospholipids is associated with ER stress and lipotoxicity in palmitate treated hepatic cells. J Lipid Res 2014; 55: 1478-1488.

[33] Boyce M, Yuan J. Cellular response to endoplasmic reticulum stress: a matter of life or death. Cell Death Differ 2006; 13: 363-373.

[34] Lin JH, Li H, Yasumura D, Cohen HR, Zhang C, Panning B, et al. IRE1 signaling affects cell fate during the unfolded protein response. Science 2007; 318: 944-949.

[35] van Schadewijk A, van't Wout EF, Stolk J, Hiemstra PS. A quantitative method for detection of spliced X-box binding protein-1 (XBP1) mRNA as a measure of endoplasmic reticulum (ER) stress. Cell Stress Chaperones 2012; 17: 275-279.

[36] Hirota M, Kitagaki M, Itagaki H, Aiba S. Quantitative measurement of spliced XBP1 mRNA as an indicator of endoplasmic reticulum stress. J Toxicol Sci 2006; 31: 149-156.

[37] Lee AS. The glucose-regulated proteins: stress induction and clinical applications. Trends Biochem Sci 2001; 26: 504-510.

[38] Hendershot LM. The ER function BiP is a master regulator of ER function. Mt Sinai J Med 2004; 71: 289-297.

[39] Lee AS. The ER chaperone and signaling regulator GRP78/BiP as a monitor of endoplasmic reticulum stress. Methods 2005; 35: 373-381. 